\title{
Computational problems in modeling arcs
}

Jim Norem, and Zeke Insepov

Citation: AIP Conference Proceedings 1777, 050003 (2016); doi: 10.1063/1.4965626

View online: http://dx.doi.org/10.1063/1.4965626

View Table of Contents: http://aip.scitation.org/toc/apc/1777/1

Published by the American Institute of Physics

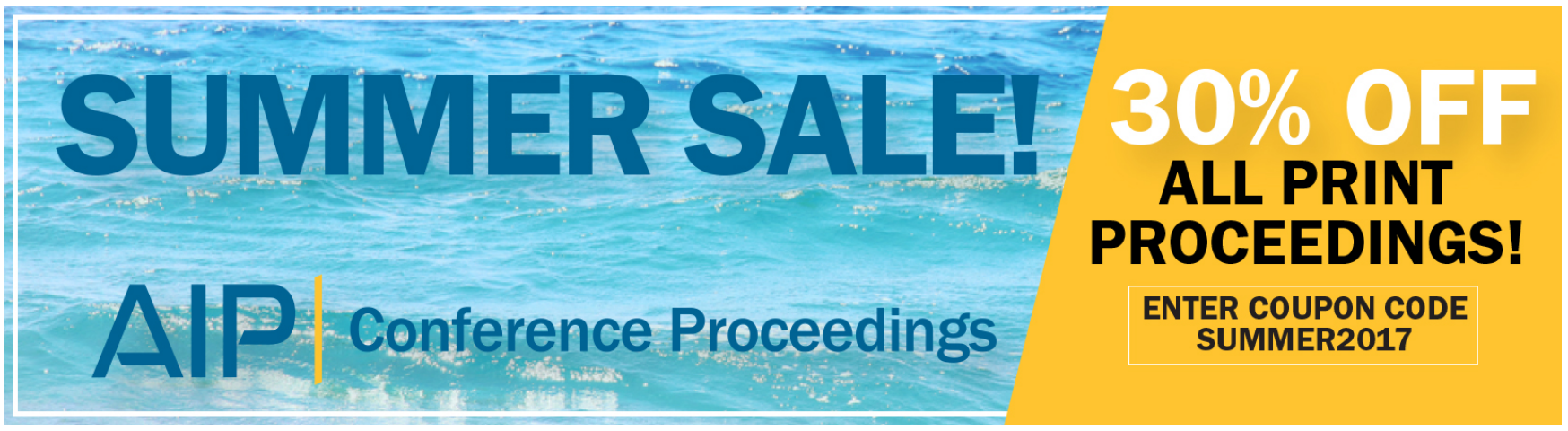




\title{
Computational Problems in Modeling Arcs
}

\author{
Jim Norem ${ }^{1, \text { a) }}$ and Zeke Insepov ${ }^{2,3, \text { b) }}$ \\ ${ }^{1}$ Nano Synergy, Inc., Downers Grove, IL 60516 \\ ${ }^{2}$ Purdue University, West Lafayette, IN 47907 \\ ${ }^{3}$ Nazarbayev University, Astana Kazakhstan

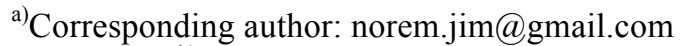 \\ b) zinsepov@purdue.edu
}

\begin{abstract}
We explore the reasons why there seems to be no common model for vacuum arcs, in spite of the importance of the field and the level of effort expended over more than one hundred years.
\end{abstract}

\section{WHY IS PROGRESS SO SLOW?}

While vacuum arcs were first identified around 1900, by Michelson and Millikan ${ }^{1,2}$ (before they even had a vacuum pump), the first reasonable explanation of the phenomenon was proposed by Lord Kelvin in $1904^{3}$, and the field has been continuously active since then, there has been no general agreement on the nature of these arcs that has developed in the past $110+$ years. There are a number of both experimental and theoretical problems that have retarded progress.

Experimentally, the arcs develop very fast and rapidly obliterate the surface defects that presumably help trigger the arcs, and the rapid arc development, over many orders of magnitude in many parameters, presents a problem with diagnostics, which tend to be useful over a comparatively small parameter range. In addition, arcing tends to occur unpredictably, both in location and time, frequently in locations (tokamaks, accelerators, etc. where access is poor) presenting further diagnostic problems. Finally, there is a wide variety of arcs and related phenomena that may or may not be related to basic arc mechanisms, for example in micrometeorite impacts with satellites, tokamak plasma/limiter interactions, the rf accelerator limits we are familiar with, laser ablation, and other applications. Even within a specific type of arc, the experimental parameters, (stored energy, pulse length, geometry, materials) would be expected to vary from one experiment to another. An additional problem, however is the complexity and variety of the mechanisms involved, which seem to operate over parameter ranges of many orders of magnitude and a diversity of environments ${ }^{4}$.

We have found that in order to model arcs, it seems necessary to incorporate many highly specific and somewhat incompatible calculations and numerical methods, for example Particle In Cell (PIC) codes to study the initial stages of the arc, and Molecular Dynamics (MD) to look at the properties of the fully developed arc ${ }^{5,6}$. Surface effects are also an integral part of this problem, however surface structure and surface issues are not generally considered a part of the plasma physics of arcs. Computational methods assume specific boundary conditions, different timescales and different internal dynamics, and it is not obvious that they are simply compatible. We find that the concept of the unipolar arcs seems to have wide applicability, however the application of these methods to his problem is not straightforward and the literature on these arcs is not well developed or unambiguous. Application of MD methods is a slow process; a recent paper looking at the application of MD to the properties of the dense (non-ideal) plasma sheath seems to be the first that makes definite predictions of the surface physics at the edge of these non-ideal plasmas, however it is not clear how to experimentally verify these predictions ${ }^{6}$. 


\section{MODELING PHYSICAL MECHANISMS IN ARCING}

In accelerator RF systems, arcs have dimensions measured in mm, survive for times on the order of 10 ns to 100 $\mu \mathrm{s}$, and involve energies measured in Joules. The mechanisms operating within arcs operate on much different spatial, time and energy scales, and the parameters of the individual mechanisms determine the type of numerical analysis that can be done.

Vacuum arcing seems to be dominated by a number of quite different mechanisms, representing different fields of study. For example, the arc seems to be described by plasma physics, however the initial breakdown stages are a complicated mixture of field emission (quantum mechanics) electrostatics, atomic physics, mechanical properties, and fracture mechanics. The surface damage that seems to determine the location of mechanical failure seems to be a result of hydrodynamics and thermodynamics. External factors also control many aspects of the arc, in particular its initiation and duration. Thus, we do not expect to see both simplicity and precision in initial modeling results.

There are a number of general methods involved in arc studies and we can explore the properties and limitations of each of them. While they do not present insolvable computational problems, they require a set of basic assumptions to unite an array of analyses of specific mechanisms.

\section{Particle in Cell}

Classical plasma physics represents the core of the arcing problem. Particle in cell calculations are a well understood method of modeling plasma physics for linear systems with two body collisions, finite numbers of particles, timescales on the order of a few nanoseconds and geometrical dimensions on the order of $10^{-5} \mathrm{~m}$. We have used PIC codes to model the first few nanoseconds of the arc development, when the arc satisfies the constraints up to the limit where we do not trust simple expressions like the derivation of the Debye length, $\lambda_{\mathrm{D}}$.

On the other hand it is not always clear how precise PIC code predictions are when the densities become large enough so that the basic assumptions of classical plasma physics no longer apply, in this case because the density of the plasma is too large to assume that the energy of the system is entirely kinetic, or the collisions are entirely between two particles.

\section{Molecular Dynamics}

We have used MD to model the mechanical failure of asperities that trigger breakdown, the self-sputtering off of solid and liquid surfaces that ultimately fuel the arc, and the properties of the sheath for dense plasmas where the assumptions of two body interactions no longer apply. Computational limitations imply timesteps for these calculations on the order of $10^{-18}-10^{-17} \mathrm{~s}$ and equilibrium times of $10^{-13} \mathrm{~s}$, over geometrical dimensions of $10^{-9} \mathrm{~m}$, many orders of magnitude smaller than the dimensions of PIC calculations ${ }^{6}$. On the other hand, MD calculations can be used when the densities are high enough so that the total electrostatic energy of the system is comparable to the kinetic energy of the particles (the nonideality parameter, $\Gamma=$ electrostatic/kinetic energy $\sim 0.5)^{6}$. MD calculations are not, in principle, compatible with PIC calculations, thus we use them to define boundary conditions and evaluate sputtering coefficients rather than to describe the evolution of the system as a whole

\section{Various mesoscale thermodynamics and other methods}

A number of other mechanisms also contribute to arcing with their own parameter ranges and variables. Cooling and development of a rough surface is a particularly complex problem.

\section{NEED FOR REALISTIC MODELING}

Since the problem of arcs has been studied for over 100 years, a simple explanation of the physics of this phenomenon seems overdue. Beyond this, arcing and gradient limits in general are a significant constraint on many aspects of technological progress and ignorance of the primary mechanisms may be associated with significant costs. We find that many of the models in print do not seem to agree with current data, and can be incompatible with basic assumptions and modern modeling. 
There are a variety of phenomema that seem to require simple explanations, such as the "chicken track" damage left by tokamak arcs, the voltage spikes produced during micrometeorite impacts on satellites and the sensitivity of RF gradient limits to strong magnetic fields, and while it might seem desirable to have a complete numerical model of arcing, the complexity of this problem seems to preclude this.

On the other hand, a complete understanding of the individual mechanisms involved, with numerical models that produce reasonable estimates of the critical variables, seems to be something that can realistically be done $\mathrm{s}^{5}$ With a basic knowledge of the scale of parameters like the Debye length, sheath potential and field emission, it is possible to generate explanations for self quenching of unipolar arcs, and other phenomena ${ }^{6}$. Likewise, studies of the cooling of thin liquid surfaces can begin to explain the range of surface damage seen in arcing. While a complete numerical model of the arc is difficult, understanding experimental data may be much easier.

\section{CONCLUSIONS}

Because of the dynamic ranges involved, complete computational methods used to model arcing from knowledge of the basic mechanisms are inherently problematic, because of widely different timescales, spatial volumes and plasma parameters. Thus modeling of arcing cannot, at present, be done using a single computational model, and must essentially be a parameter list obtained from a variety of models each applying to one mechanism. This requires some interpretation to produce a general picture of arcing, and a "complete" model of arcing becomes a series of calculations of specific mechanisms together with a general picture and set of assumptions that tie the mechanisms together. On the other hand, there seems to be sufficient knowledge of the individual mechanisms involved to produce useful predictions and explanations of experimental data.

\section{ACKNOWLEDGEMENTS}

This paper summarizes many years study and many papers (see Ref 5 and 6 and references in these papers). We are indebted to the help of our co-authors on the many papers referred to, and to the support of the USDOE, the Muon Program and the operating staff at the Fermilab Muon Test Area, where the data that inspired this work was produced.

\section{REFERENCES}

1. R.F. Earhart, Philos. Mag. 1, 147 (1901).

2. R. A. Millikan, Autobiography, Prentice-Hall, New York (1950).

3. Lord Kelvin, Phil. Mag. 8, 534 (1904). Also, Mathematical and Physical Papers, Vol. VI, Voltaic theory, Radioactivity, Electrions, Navigation and Tides, Miscellaneous, Cambridge University Press, Cambridge (1911), p. 211.

4. A. Anders, Cathodic Arcs: From Fractal Spots to Energetic Condensation, Springer, New York (2008).

5. Z. Insepov and J. Norem, J. Vac. Sci Technol. A 31, 011302 (2013).

6. I. V. Morozov, G. E. Norman, Z. Insepov, and J. Norem, Phys. Rev. ST Accel. Beams 15, 053501 (2012). 